\section{Multifrequency invisibility and masking of cylindrical dielectric objects using double-positive and double-negative metamaterials}

To cite this article: A E Serebryannikov and Ekmel Ozbay 2009 J. Opt. A: Pure Appl. Opt. 11114020

View the article online for updates and enhancements.
Related content

- Plasmonic and metamaterial cloaking:
physical mechanisms and potentials
Andrea Alù and Nader Engheta
- Plasmonic cloaking of cylinders: finite
$\frac{\text { length, oblique illumination and cross- }}{\text { polarization coupling }}$
Andrea Alù, David Rainwater and Aaron
Kerkhoff
- Transformation optics for the full dielectric
electromagnetic cloak and metal-dielectric
planar hyperlens
D P Gaillot, C Croënne, F Zhang et al.

\section{Recent citations}

- Magnetically controlled multifrequency
$\frac{\text { invisibility cloak with a single shell of ferrite }}{\text { Material }}$
Xiaohua Wang and Youwen Liu
$\frac{\text { Invisibility and Cloaking Based on }}{\text { Scattering Cancellation }}$
- Elec-Yen Chen et al
$\frac{\text { ferroelectric cloak }}{\text { Peining Li et al }}$




\title{
Multifrequency invisibility and masking of cylindrical dielectric objects using double-positive and double-negative metamaterials
}

\author{
A E Serebryannikov and Ekmel Ozbay \\ Nanotechnology Research Center-NANOTAM, Bilkent University, 06800 Ankara, Turkey, \\ Department of Physics, Bilkent University, 06800 Ankara, Turkey \\ and \\ Department of Electrical and Electronics Engineering, Bilkent University, 06800 Ankara, \\ Turkey \\ E-mail: andriy@bilkent.edu.tr (A E Serebryannikov)
}

Received 17 January 2009, accepted for publication 19 March 2009

Published 17 September 2009

Online at stacks.iop.org/JOptA/11/114020

\begin{abstract}
We demonstrate that a circular dielectric cylinder can be nearly invisible at multiple frequencies when being coated with a ring shell, which is made of an isotropic material simultaneously showing large positive or large negative values of permittivity and permeability. The suggested cloaking mechanism is based on the use of radial resonances, which are similar to those in conventional Fabry-Perot resonators. It can be used for cylindrical objects for a wide range of variation of the diameter-to-wavelength ratio, which includes the values corresponding to subwavelength to resonant-sized objects. The presence of frequency dispersion of the shell material positively affects the possibility of multifrequency operation.
\end{abstract}

Keywords: cloaking, masking, Fabry-Perot resonator, Drude-Lorentz materials

(Some figures in this article are in colour only in the electronic version)

\section{Introduction}

The possibility of the cloaking of dielectric and metallic objects has been the focus of interest throughout the past three years. Two main classes of cloaking approaches can be distinguished, depending on whether it is obtained in a region that is external or internal to the cloaking body [1]. The former is connected to the resonant interaction based on the anomalous localized resonances, allowing for the properly located dipole-type sources to be made invisible in an external region [2, 3]. The latter can be realized by using several approaches, according to which the impinging field is re-routed around the covered object in such a manner that it is not seen (ideal cloaking) or seen poorer (non-ideal cloaking) by a far-field observer and even by a near-field observer, see, e.g., [4-10].

The coordinate transformation approach has been suggested by Pendry et al for obtaining a metamaterial shell, which would exclude electromagnetic fields from the covered object without affecting the exterior fields [4]. The required radial dependence of anisotropic relative permittivity and permeability, $\varepsilon_{\rho}=\mu_{\rho}, \varepsilon_{\phi}=\mu_{\phi}$ and $\varepsilon_{z}=\mu_{z}$, and the possible applicability to a wide class of problems under a rather arbitrary wavelength condition belong to the basic features of this approach. For example, it has successfully been applied to the design of a microwave cloak for a PEC circular cylinder [5].

At optical frequencies, magnetism has been demonstrated in metamaterials by several research groups [11-16], but the designed structures still show relatively high losses. If the magnetic field is polarized along the axial direction, an optical cloak can be created without any magnetism, so that $\varepsilon_{\rho}$ only depends on the radial coordinate [9]. Various cloak performances have been suggested, which include in particular those using wires made of a polaritonic material, concentric silicon photonic crystal layers, metamaterials based on metallic 
split-ring resonators or cut wires, and multilayer plasmonic metamaterials, see, e.g., [10, 17-20].

The non-resonant approach based on the use of single- and double-layer shells made of isotropic homogeneous plasmonic materials or metamaterials has been suggested in $[6,7,21,22]$. In particular, it has been shown that the cloaking can be realized at least for two frequencies simultaneously, provided that the shell consists of two concentric plasmonic layers [21, 22]. Until now, this mechanism has been demonstrated for subwavelength and moderately sized objects.

In the present paper, we study theoretically the potential of an alternative approach in order to achieve multifrequency cloaking, which is based on the use of shells made of artificial materials with simultaneously large positive or large negative permittivity and permeability. In contrast to most of the recent studies, we will consider a wide range of frequency variation, which is extended at least from $D / \lambda=1 / 6$ to 1 , where $D$ is the diameter of the coated cylinder. It will be shown that several frequencies at least can exist simultaneously for which the scattering cross section is dramatically reduced. Consideration will be restricted to circular cylinders which are made of moderate-permittivity dielectrics. While the main attention in the field of metamaterials has been paid to the operational regimes with a relatively small negative index of refraction, simultaneously large positive or large negative values of permittivity and permeability can be obtained for the same performance.

The exploited physical mechanism is related to the radial resonances within the coating shells, which can be roughly interpreted by using the analogy with conventional Fabry-Perot resonators. The main goal of the present paper is to demonstrate the principal possibility of achieving a multifrequency reduction of the scattering cross section owing to multiple radial resonances. Near- and farfield characteristics will firstly be studied for hypothetical dispersion-free matched and mismatched materials, and then the main effects will be validated for materials showing DrudeLorentz dispersion.

\section{Background}

The geometry of the problem is shown in figure 1 . The dielectric cylinder, which is covered by the ring shell that is made of a metamaterial, is illuminated by a TE or TM polarized electromagnetic wave. The axial field $\left(f_{z}=H_{z}\right.$ for TE polarization and $f_{z}=E_{z}$ for TM polarization) is given by

$$
f_{z}=\sum_{n=-\infty}^{\infty}(-\mathrm{i})^{n}\left[J_{n}(k \rho)+c_{n} H_{n}^{(2)}(k \rho)\right] \exp (\mathrm{i} n \phi)
$$

at $\rho>R$,

$$
f_{z}=\sum_{n=-\infty}^{\infty}(-\mathrm{i})^{n}\left[b_{n}^{(1)} J_{n}\left(k_{\mathrm{s}} \rho\right)+b_{n}^{(2)} Y_{n}\left(k_{\mathrm{s}} \rho\right)\right] \exp (\mathrm{i} n \phi)
$$

at $R>\rho>r$ and

$$
f_{z}=\sum_{n=-\infty}^{\infty}(-\mathrm{i})^{n} a_{n} J_{n}\left(k_{\mathrm{c}} \rho\right) \exp (\mathrm{i} n \phi)
$$

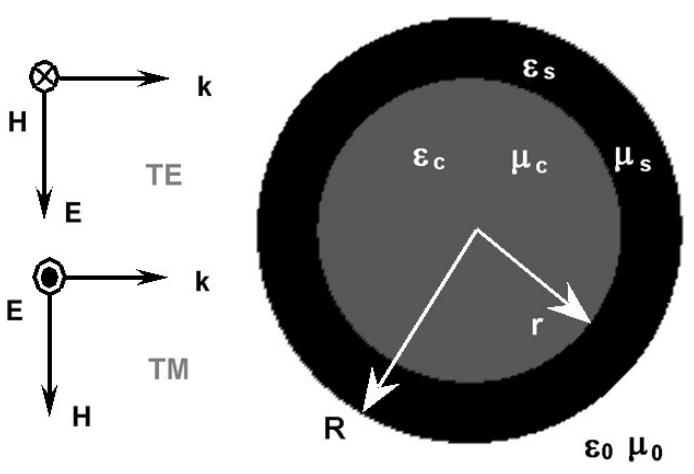

Figure 1. Geometry of the studied problem.

at $\rho<r$, where $k_{\mathrm{s}}=k \sqrt{\varepsilon_{\mathrm{s}}} \sqrt{\mu_{\mathrm{s}}}, k_{\mathrm{c}}=k \sqrt{\varepsilon_{\mathrm{c}}} \sqrt{\mu_{\mathrm{c}}}$ and $k=\omega / \mathrm{c}$. Analytical expressions for $a_{n}, b_{n}^{(1)}, b_{n}^{(2)}$ and $c_{n}$ were derived using the conservation of the tangential field components at $\rho=r, R$ and used in near- and far-field analysis. The normalized scattering cross section is calculated as follows:

$$
\sigma=(k R)^{-1} \sum_{n=-\infty}^{\infty} c_{n}^{2}
$$

The suggested approach to reduce $\sigma$ is based on the use of Fabry-Perot-type radial resonances. Its basic idea can be understood by using the analogy with conventional FabryPerot resonators. The total transmission occurs in such a resonator, meaning in fact that a far-zone observer located in the transmission half-space does not see it at multiple equidistant frequencies. Because of the shell curvature, this analogy might inappropriately describe the dominant physics of the expected reduction of the scattering cross section. However, if the cylinder radius is much larger than the in-material wavelength, it could be qualitatively correct. Therefore, this approach requires the use of materials with a high index of refraction and, hence, with strong frequency dispersion. The cloaking is considered to be ideal if $\sigma \equiv 0$ and non-ideal if $\sigma \approx 0$. To measure the extent to which it is nonideal, a comparison of the $\sigma$ values that are obtained for the same object with and without a shell is often used. According to [23], a strong reduction of $\sigma$ is called masking.

At microwave frequencies, there are various possibilities for obtaining simultaneously large positive or large negative values of $\operatorname{Re} \varepsilon$ and $\operatorname{Re} \mu$. In particular, strong magnetism can be obtained using split-ring resonators, some nanocomposites [24, 25] and photonic crystals containing ferroelectric constituents [26], which might solely show the very large values of $\operatorname{Re} \varepsilon$. Values of $\operatorname{Re} \mu$ from 10 to several hundreds have been reported. Simultaneously large $\operatorname{Re} \varepsilon$ and $\operatorname{Re} \mu$ (up to 10) can be obtained in isotropic artificial materials that are composed of resonant spheres [27]. Large values of the index of refraction (e.g. $N=5.51$ ) have recently been demonstrated for the metamaterials constructed by using metallic gratings with periodic subwavelength slits [28].

In addition to the works [11-16], which are dedicated to metamaterials, one should mention methods of obtaining magnetism at optical frequencies such as those based on the use of plasmonic crystals composed of nanorods with a large 

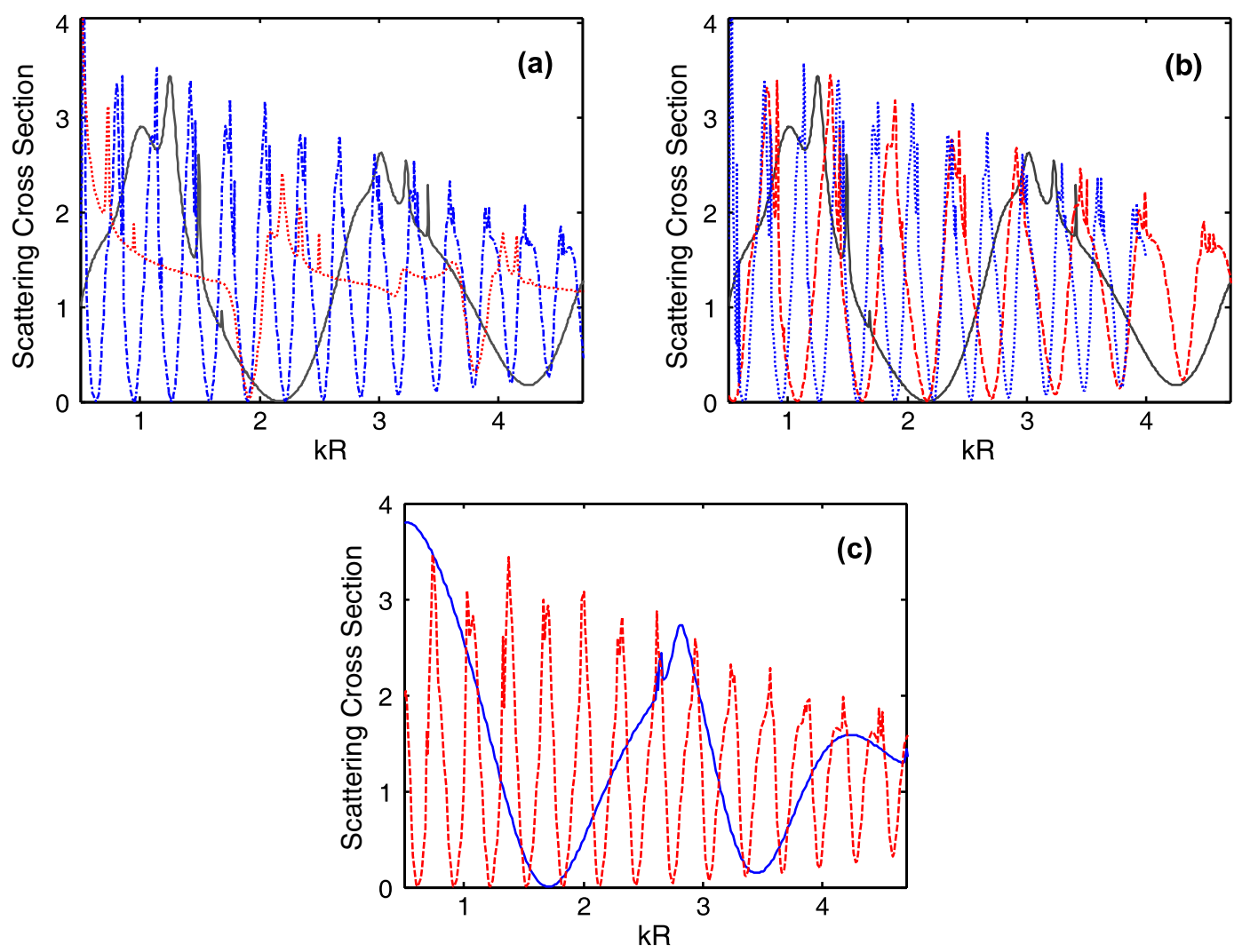

Figure 2. Scattering cross section versus $k R$ at $\varepsilon_{\mathrm{s}}=\mu_{\mathrm{s}}=5.8$ (solid line), $\varepsilon_{\mathrm{s}}=\mu_{\mathrm{s}}=35.4$ (dashed-dotted line), and $\varepsilon_{\mathrm{s}}=35.4$ and $\mu_{\mathrm{s}}=1$ (dotted line) for TE polarization-plot (a); $\varepsilon_{\mathrm{s}}=\mu_{\mathrm{s}}=5.8$ (solid line), $\varepsilon_{\mathrm{s}}=\mu_{\mathrm{s}}=21$ (dashed line) and $\varepsilon_{\mathrm{s}}=\mu_{\mathrm{s}}=35.4$ (dotted line) for TM polarization—plot (b); $\varepsilon_{\mathrm{s}}=\mu_{\mathrm{s}}=-5.8$ (solid line) and $\varepsilon_{\mathrm{s}}=\mu_{\mathrm{s}}=-35.4$ (dashed line) for TM polarization-plot $(\mathrm{c}) ; \varepsilon_{\mathrm{c}}=\mu_{\mathrm{c}}=1$ and $R / r=1.4$.

diameter-to-lattice-constant ratio [29], metal nanoclusters [30] and dielectrics with strong anisotropy [31]. Although the achieving of strong magnetism with low losses at optical frequencies is still a challenging task, the state-of-the-art and existing trends in the field of metamaterials look promising for the realization of the suggested approach. The parameters for simulations will be chosen by taking them into account.

\section{Results and discussion}

\subsection{Empty impedance-matched shells}

Figure 2 shows the conditions at which $\sigma \approx 0$ can be obtained for an empty shell at several frequencies simultaneously. In figure 2(a), the results are presented for TE polarization and a shell made of a double-positive material with $Z_{\mathrm{s}}=Z_{0}=Z_{\mathrm{c}}$, where $Z_{0}, Z_{\mathrm{s}}$ and $Z_{\mathrm{c}}$ mean the impedances of free space and the materials of the shell and core, respectively. This case is similar in some sense to Pendry's cloak, since $\varepsilon_{\mathrm{s}}=\mu_{\mathrm{s}}$. However, in our case $\varepsilon_{\mathrm{s}}$ and $\mu_{\mathrm{s}}$ show no radial variation. It is seen that $\sigma \approx 0$ can be obtained at several frequencies if $\varepsilon_{\mathrm{s}}$ and $\mu_{\mathrm{s}}$ are simultaneously large positive. The increase of $\varepsilon_{\mathrm{s}}=\mu_{\mathrm{s}}$ from 5.8 to 35.4 results in that at least eight such frequencies occur instead of a sole frequency within the considered range. In turn, a high-permittivity non-magnetic shell with $\varepsilon_{\mathrm{s}}=35.4$ and $\mu_{\mathrm{s}}=1$ (now $Z_{\mathrm{s}} \neq Z_{0}$ ) also allows obtaining $\sigma \approx 0$ at a sole frequency only.
At $\varepsilon_{\mathrm{s}}=\mu_{\mathrm{s}}=35.4$, the minima of $\sigma$ are nearly equidistant and located for $0.5<k R<3$ at $k R=0.63,0.95,1.27$, $1.59,1.90,2.22,2.53$ and 2.85 . The equidistance is typical for the cavity volume modes. Furthermore, the observed minima approximately satisfy the condition

$$
R-r=m \lambda_{\mathrm{s}} / 2
$$

where $m=2,3,4, \ldots, \lambda_{\mathrm{s}}=\lambda /\left|\sqrt{\varepsilon_{\mathrm{s}}} \sqrt{\mu_{\mathrm{s}}}\right|$ and $\lambda$ is the freespace wavelength, so that any two neighboring minima differ by $\lambda_{\mathrm{s}} / 2$. The same features are observed for TM polarization, see figure 2(b). Owing to the reciprocity, $\sigma^{\mathrm{TE}}=\sigma^{\mathrm{TM}}$ at $\varepsilon_{\mathrm{s}}=$ $\mu_{\mathrm{s}}$ and $\varepsilon_{\mathrm{c}}=\mu_{\mathrm{c}}=1$. Therefore, the results for $\varepsilon_{\mathrm{s}}=\mu_{\mathrm{s}}=35.4$ in figures 2(a) and (b) coincide. The same remains true for the results for $\varepsilon_{\mathrm{s}}=\mu_{\mathrm{s}}=5.8$. At $\varepsilon_{\mathrm{s}}=\mu_{\mathrm{s}}=21$, the minima of $\sigma$ appear at $k R=0.54,1.08,1.625,2.165$ and 2.705. This is in agreement with equation (5).

Figure 2(c) presents $\sigma$ versus $k R$ at $\varepsilon_{\mathrm{s}}=\mu_{\mathrm{s}}<0$. As can be expected, the smaller the $\varepsilon_{\mathrm{s}}$, the more the extrema of $\sigma$ are located within a fixed $k R$ range. Hence, $\varepsilon_{\mathrm{s}}$ and $\mu_{\mathrm{s}}$ can be either simultaneously large positive or large negative in order to obtain $\sigma \approx 0$ at several frequencies. At $\varepsilon_{\mathrm{s}}=\mu_{\mathrm{s}}=-35.4$, $\sigma \approx 0$ for $k R=0.61,0.915,1.22,1.525,1.83,2.135,2.44$ and 2.745 . The minima are nearly equidistant but shifted with respect to those in figures 2(a) and (b) at $\varepsilon_{\mathrm{s}}=\mu_{\mathrm{s}}=35.4$.

The observed behavior of the transmission shows the analogy with that in conventional Fabry-Perot resonators. 

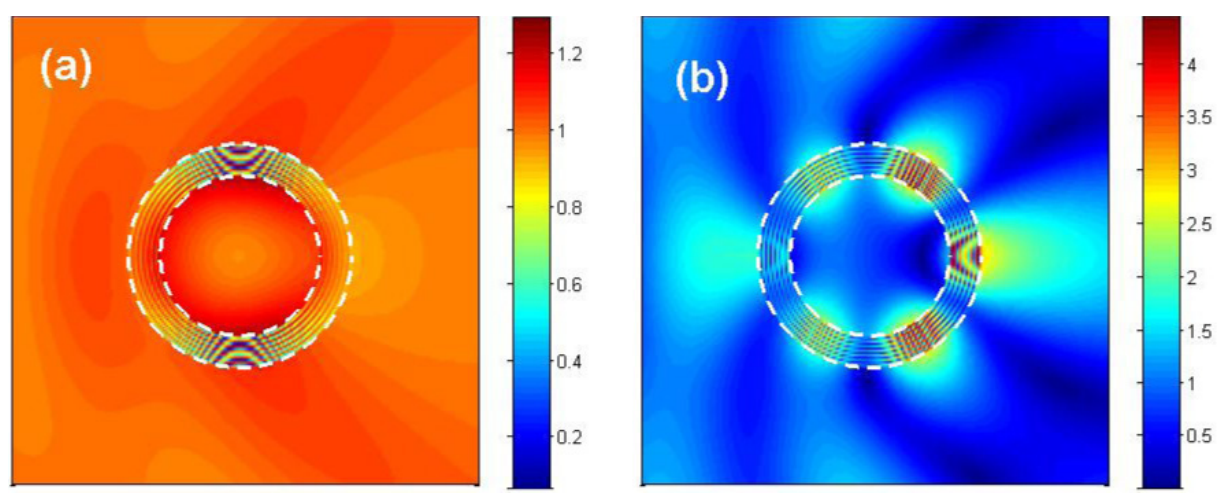

Figure 3. Axial magnetic field for TE polarization at $\varepsilon_{\mathrm{s}}=\mu_{\mathrm{s}}=35.4, \varepsilon_{\mathrm{c}}=\mu_{\mathrm{c}}=1, R / r=1.4$, and $k R=1.9$ (a) and $k R=2.044$ (b).
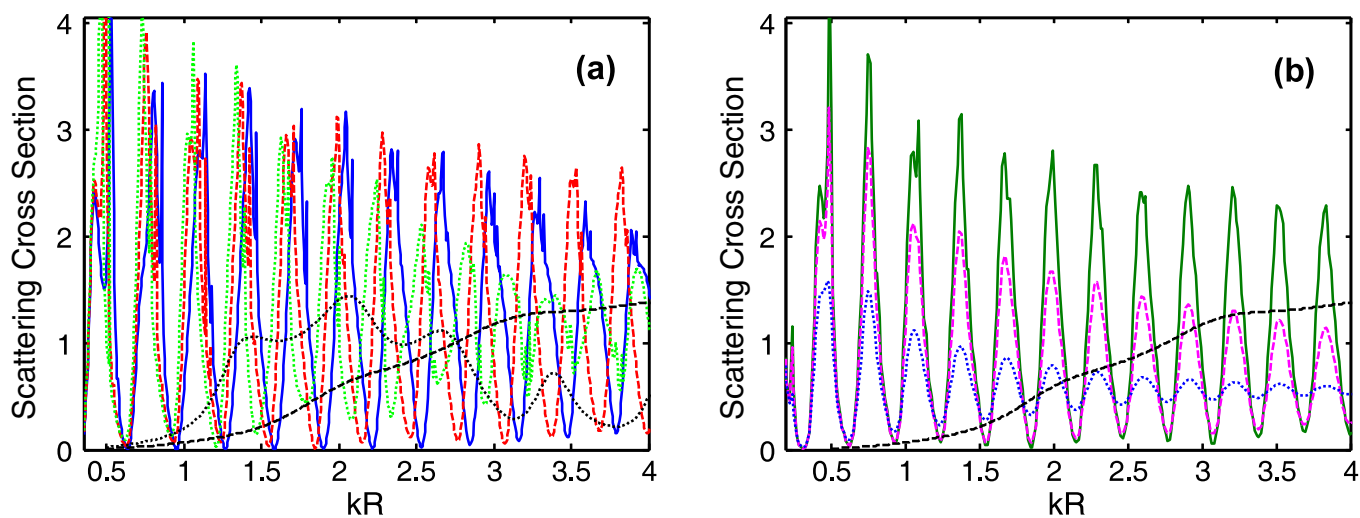

Figure 4. Scattering cross section versus $k R$ at $\mu_{\mathrm{c}}=1$ and $R / r=1.4$ for TE polarization: $\varepsilon_{\mathrm{c}}=1$ (solid line), $\varepsilon_{\mathrm{c}}=2.8$ (dashed lines) and $\varepsilon_{\mathrm{c}}=5.8$ (dotted lines); $\varepsilon_{\mathrm{s}}=\mu_{\mathrm{s}}=35.4$-lines with multiple strong extrema and $\varepsilon_{\mathrm{s}}=\mu_{\mathrm{s}}=1$-lines without multiple extrema-plot (a); $\varepsilon_{\mathrm{c}}=2.8, \operatorname{Im} \varepsilon_{\mathrm{s}} / \operatorname{Re} \varepsilon_{\mathrm{s}}=0.001$ (solid line), $\operatorname{Im} \varepsilon_{\mathrm{s}} / \operatorname{Re} \varepsilon_{\mathrm{s}}=0.01$ (dashed line) and $\operatorname{Im} \varepsilon_{\mathrm{s}} / \operatorname{Re} \varepsilon_{\mathrm{s}}=0.04$ (dotted line); $\operatorname{Re} \varepsilon_{\mathrm{s}}=\operatorname{Re} \mu_{\mathrm{s}}=35.4$, $\operatorname{Im} \mu_{\mathrm{s}} / \operatorname{Re} \mu_{\mathrm{s}}=\operatorname{Im} \varepsilon_{\mathrm{s}} / \operatorname{Re} \varepsilon_{\mathrm{s}}$-lines with multiple extrema, $\varepsilon_{\mathrm{s}}=\mu_{\mathrm{s}}=1$-line without multiple extrema—plot (b).

Indeed, equation (5) coincides with the condition of total transmission in the resonators with the distance between the mirrors $b^{\mathrm{FP}}=R-r$, which are filled with a material that possesses the index of refraction $N^{\mathrm{FP}}=\sqrt{\varepsilon_{\mathrm{s}}} \sqrt{\mu_{\mathrm{s}}}$. Consider now the near-field patterns at the extrema. Figure 3 shows a typical example of a magnetic field in the TE case for a minimum and a maximum of $\sigma$ from figure 2. Figure 3(a) corresponds to a minimum. One can see that $\left|H_{z}\right|$ varies slightly beyond the shell. At the same time, $\left|H_{z}\right|$ shows several variations in the radial direction in the shell, being in agreement with the theory of Fabry-Perot resonators. Here, $\sigma_{\min } \approx 3.5 \times 10^{-3}$ and $R-r \approx 3 \lambda_{\mathrm{s}}$. In contrast, the field patterns at the maxima of $\sigma$ are associated with the whisperinggallery modes, see figure 3(b). They correspond to the waves, which are slow in the azimuthal direction $(k R / l<1, l$ is azimuthal mode index, $l=3$ ). The reciprocity in the near-field patterns manifests itself in that $\mathrm{TE} \leftrightarrow \mathrm{TM}$ and $\mathbf{H} \leftrightarrow \mathbf{E}$, so that figures 3(a) and (b) also correspond to the $E_{z}$ component for TM polarization.

\subsection{Dielectric object inside a matched shell}

Now, we will show that the multiple frequencies with $\sigma \approx 0$ can remain for similar structures as in section 3.1, if the shell interior is filled with a moderate- $\varepsilon$ dielectric. Now, $Z_{\mathrm{s}}=Z_{0}$ and $Z_{\mathrm{c}} \neq Z_{\mathrm{s}}$, i.e. the shell is matched with free space but mismatched with the interior. An example of the scattering cross section is presented in figure 4(a) for TE polarization. Here, the case corresponding to the dashed-dotted line in figure 2(a) is shown together with two other cases, in which the core has $\varepsilon_{\mathrm{c}}>1$. Besides, $\sigma$ is presented for the corresponding non-coated cylinders. The possibility of a substantial reduction of the scattering cross section is clearly seen. Due to the dielectric filling, the extrema are shifted towards smaller $k R$. For example, the minima appear at $k R=1.58$ for $\varepsilon_{\mathrm{c}}=1$, $k R=1.54$ for $\varepsilon_{\mathrm{c}}=2.8, k R=1.465$ for $\varepsilon_{\mathrm{c}}=5.8$ and at $k R=1.9$ for $\varepsilon_{\mathrm{c}}=1, k R=1.844$ for $\varepsilon_{\mathrm{c}}=2.8$ and $k R=1.788$ for $\varepsilon_{\mathrm{c}}=5.8$. This behavior qualitatively coincides with that predicted by the perturbation theory of the cavity resonators, since bringing a dielectric body into a cavity results in a decrease of the resonance frequency. Generally, an increase of $\varepsilon_{\mathrm{c}}$ leads to the number of frequencies, at which $\sigma \approx 0$, decreasing and the corresponding $k R$ range narrowing. It is worth noting that $\sigma_{\min } / \sigma^{\text {nc }} \approx 1 / 45$ for $k R=1.844$ and $\sigma_{\min } / \sigma^{\mathrm{nc}} \approx 1 / 10$ in the vicinity of $k R=2.14$ at $\varepsilon_{\mathrm{c}}=2.8$, where $\sigma^{\text {nc }}$ is the scattering cross section of the non-coated cylinder. For TM polarization, similar far-field effects are observed. As has been shown above, $\sigma(\omega)$ is not changed with the change of polarization at $\varepsilon_{\mathrm{c}}=1$. While the effect of the core with $\varepsilon_{\mathrm{c}}>1$ appears in a perturbation-like way, the 

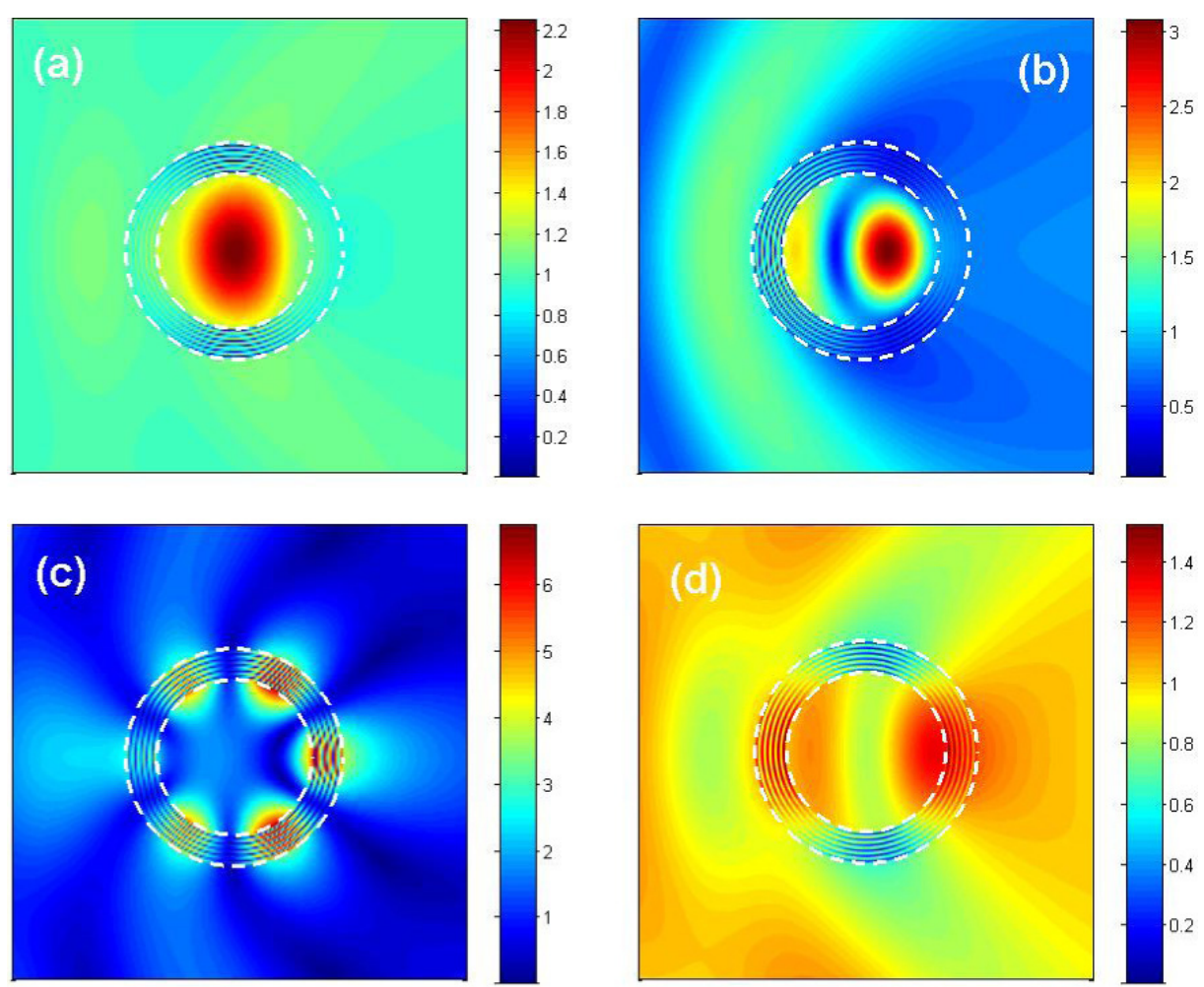

Figure 5. Axial magnetic field for TE polarization at $k R=1.844$ (a), $k R=1.788$ (b) and $k R=1.706$ (c), and axial electric field for TM polarization at $k R=1.83(\mathrm{~d}) ; R / r=1.4, \varepsilon_{\mathrm{s}}=\mu_{\mathrm{s}}=35.4, \mu_{\mathrm{c}}=1$, and $\varepsilon_{\mathrm{c}}=2.8((\mathrm{a}),(\mathrm{c}),(\mathrm{d}))$ and $\varepsilon_{\mathrm{c}}=5.8(\mathrm{~b})$.

difference between the dependences of $\sigma$ on $k R$ for TE and TM polarizations can be relatively weak. The main difference is related to the extent to which the minima are shifted comparing to the case with $\varepsilon_{\mathrm{c}}=1$.

Until now, it has been assumed that the shell material is lossless. Figure 4(b) shows $\sigma$ versus $k R$ for three shells, which differ from that in figure 4(a) in that now $\operatorname{Im} \varepsilon_{\mathrm{s}} \neq 0$ and $\operatorname{Im} \mu_{\mathrm{s}} \neq 0$. Note that the solid line in figure 4(b) nearly coincides with a dashed line in figure 4(a) for which $\operatorname{Im} \varepsilon_{\mathrm{s}}=0$. The effect of the losses is weak at $k R<1.25$, i.e. where either no reduction or a weak reduction of $\sigma$ does appear. At larger $k R$, the minima become weaker once $\operatorname{Im} \varepsilon_{\mathrm{s}}$ is increased, and can even disappear. Nevertheless, a substantial decrease of $\sigma$ takes place due to the covering at least if $k R>1.5$ and $\operatorname{Im} \varepsilon_{\mathrm{s}} / \operatorname{Re} \varepsilon_{\mathrm{s}} \leqslant 0.01$. Although the minima locations are insensitive to the variation of $\operatorname{Im} \varepsilon_{\mathrm{S}}$ and $\operatorname{Im} \mu_{\mathrm{s}}$, the corresponding $\sigma$ values can differ significantly.

Next, we will consider the near-field patterns, which correspond to some extrema of $\sigma$. Figure 5 presents the typical examples for a minimum (plots (a), (b)) and maximum (plot (c)) of $\sigma$ in the TE case and for a minimum of $\sigma$ in the TM case (plot (d)) from figure 4(a). The extent to which $\sigma$ deviates from zero correlates well with the observed near-field features. Despite that the $k R$ values in figures 3(a) and 5(a) differ slightly, while $\sigma$ is equal to $3.5 \times 10^{-3}$ and $8 \times 10^{-3}$, respectively, the field pattern within the core is quite different. Now it looks like that of a 'locked' resonance, whose field is mainly determined by the space harmonic with $n=0$. Hence, the case of $\sigma \approx 0$ is not necessarily connected with a certain type of field pattern (i.e. resonance or non-resonance) within the interior. Keeping in mind these facts, one can conclude that all the observed near- and far-field features cannot be directly interpreted in terms of the conventional perturbation theory.

Considering the shell as an isolator should provide one with the guidelines for an appropriate interpretation. This would be reasonable since the differences between figures 2(a) and 4(a) appear within the core region only. On the other hand, the above-discussed analogy with Fabry-Perot resonators remains valid, although the media at $\rho<r$ and $\rho>R$ are different now. The larger the $\varepsilon_{\mathrm{c}}$, the stronger the $H_{z}$ inside the core should be. However, this effect can be accompanied by such a strong increase of $\sigma$ at the minimum that it cannot be more associated with the masking regime. For example, this situation occurs in figure 4(a) in the vicinity of the minimum of $\sigma$ at $k R=1.9$ and $\varepsilon_{\mathrm{c}}=1$. At $\varepsilon_{\mathrm{c}}=5.8$, it is shifted to $k R=1.788$, where $\sigma \approx 0.44$. In the latter case, the field still has a maximum inside the core, but the region of maximal $\left|H_{z}\right|$ is strongly flattened due to the compression along the abscissa axis, see figure 5(b).

It is noteworthy that often the variation of $\varepsilon_{\mathrm{c}}$ from 2.8 to 5.8 does not lead to a substantial change in the field topology within the core, despite the difference occurring in the $\sigma$ value. For example, this occurs for the minima in the vicinity of $k R=1.5$ in figure 4(a), where $\sigma \approx 0.03$ at $\varepsilon_{\mathrm{c}}=2.8$ and $\sigma \approx 0.14$ at $\varepsilon_{\mathrm{c}}=5.8$. At some minima of $\sigma$, e.g. at $k R=2.15$ for $\varepsilon_{\mathrm{c}}=2.8$ and TE polarization, the field pattern inside the core behaves in an intermediate fashion between those in figures 5(a) and (b). The near-field patterns at the maxima of $\sigma$ are associated with the whispering-gallery modes. An example is shown in figure 5(c) where $l=3$. The only difference in 


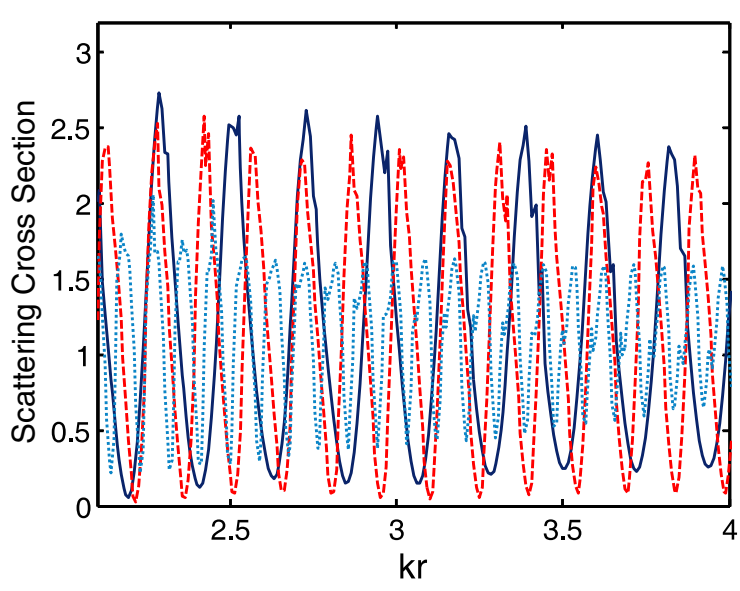

Figure 6. Scattering cross section versus $k r$ at $\varepsilon_{\mathrm{s}}=\mu_{\mathrm{s}}=35.4$, $\varepsilon_{\mathrm{c}}=2.8$ and $\mu_{\mathrm{c}}=1$ for TE polarization at $R / r=1.4$-solid line, $R / r=1.6$-dashed line and $R / r=2$-dotted line.

the comparison with figure 3(b) is that now the field is stronger within the core subregions, which are adjacent to the shell. In all the examples presented, $E_{\rho}$ is very weak within the shells, leading to isolation.

For TM polarization, values of $\left|E_{z}\right|$ at the minima of $\sigma$ in the shell, core and surrounding space differ weaker than those of $\left|H_{z}\right|$ for TE polarization, while the core subregion with the largest $\left|E_{z}\right|$ is now shifted towards larger positive $x$. Figure 5(d) presents an example corresponding to a minimum of $\sigma\left(\sigma_{\min } \approx 0.04\right)$. At the maxima of $\sigma$, we obtain the field patterns, which can again be associated with the whisperinggallery modes.

For the parameters from figures 2 and 4 , the upper boundary of the frequency range, in which $\sigma \approx 0$ at multiple frequencies, can be roughly estimated as $k r=2.2$. It can be extended owing to the optimized choice of the value of $R / r$. Figure 6 demonstrates that more than a twofold widening of this range can be achieved, provided that $R / r=1.6$ instead of $R / r=1.4$, so that the resonant-sized dielectric cylinders with $2 r / \lambda \geqslant 1$ can now be masked at least at $\varepsilon_{\mathrm{c}}=2.8$ (for example, $\sigma_{\min }<0.06$ at $k r=3.1$ and 3.25). The extent to which $\sigma$ can be reduced depends on the ratio $r /(R-r)$ non-monotonically and is sensitive to the variations of $\varepsilon_{\mathrm{c}}$ and polarization. At $\varepsilon_{\mathrm{c}}=5.8$ and for the same remaining parameters as in figure 6 , the smallest values of $\sigma_{\min }$ at $k r>2.5$ correspond to $R / r=2$. However, in this case $\sigma_{\min }>0.2$ for both polarizations, so that the extension of the range of masking up to $2 r / \lambda>1$ cannot be achieved at least for the values of $R / r$ used.

All the features observed at large positive $\varepsilon_{\mathrm{s}}$ and $\mu_{\mathrm{s}}$ remain at large negative $\varepsilon_{\mathrm{s}}$ and $\mu_{\mathrm{s}}$. Figure 7 demonstrates the effect exerted by changing the sign of $\varepsilon_{\mathrm{s}}$. It is seen that neither the number of the extrema within a wide fixed $k R$ range nor the values of $\sigma_{\min }$ are strongly affected by this change. At $k R<1$, the effect of the sign on the extrema location is vanishingly small, but the difference is increased with $k R$. At $k R<4.5$, the minima locations at $\varepsilon_{\mathrm{s}}>0$ approximately correspond to the maxima locations at $\varepsilon_{\mathrm{s}}<0$ and vice versa. This example leads one to the analogy allowing the prediction of the possible changes in the transmission spectrum, which can originate

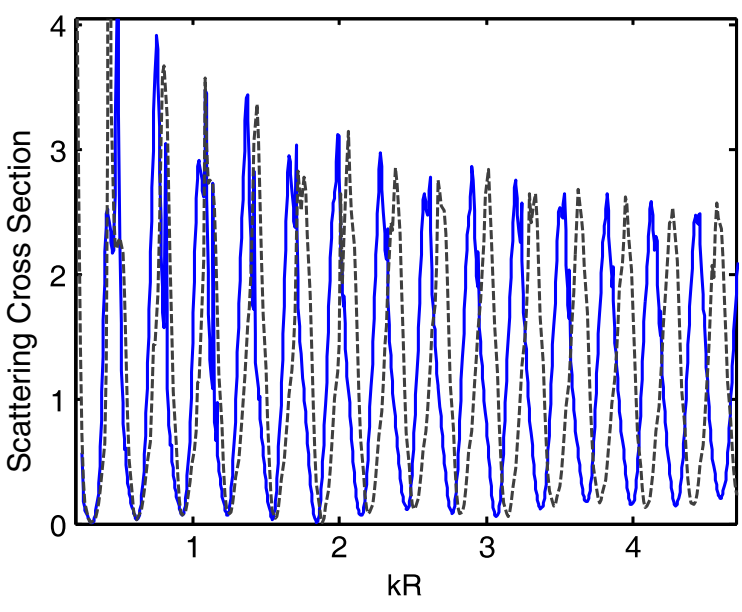

Figure 7. Scattering cross section versus $k R$ at $\varepsilon_{\mathrm{s}}=\mu_{\mathrm{s}}=35.4$ - solid line, $\varepsilon_{\mathrm{s}}=\mu_{\mathrm{s}}=-35.4$-dashed line, $\varepsilon_{\mathrm{c}}=2.8, \mu_{\mathrm{c}}=1$ and $R / r=1.4$ for TE polarization.

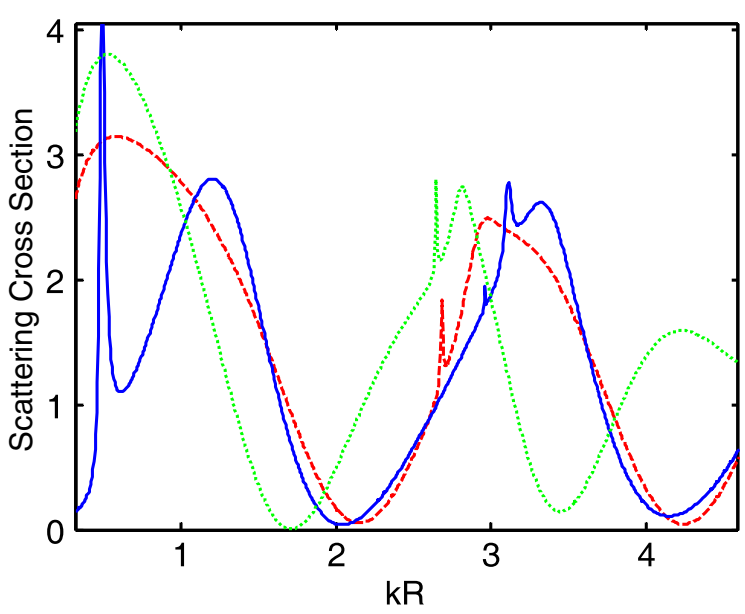

Figure 8. Scattering cross section versus $k R$ at $\varepsilon_{\mathrm{c}}=2.8$ for TE polarization-solid line, $\varepsilon_{\mathrm{c}}=2.8$ for TM polarization-dashed line and $\varepsilon_{\mathrm{c}}=1$-dotted line; $\varepsilon_{\mathrm{s}}=\mu_{\mathrm{s}}=-5.8, \mu_{\mathrm{c}}=1$ and $R / r=1.4$.

from the changing sign of the index of refraction of the filling medium in Fabry-Perot resonators.

It is shown in figure 2 that $\sigma$ can be near zero for the empty shells even at relatively small $\varepsilon_{\mathrm{s}}=\mu_{\mathrm{s}}$. This remains true at $\varepsilon_{\mathrm{c}}>1$ for both negative-index and positive-index materials of the shell. An example is shown in figure 8 for $\varepsilon_{\mathrm{s}}=\mu_{\mathrm{s}}=-5.8$. Here, the largest among the two $k R$ values with $\sigma \approx 0$ corresponds to $2 r / \lambda \approx 0.95$. The minima locations are in agreement with (5). The reciprocity manifests itself in that a simultaneous change of the sign of $\varepsilon_{\mathrm{s}}=\mu_{\mathrm{s}}$ and polarization, and the replacement of the dielectric cylinder having $\varepsilon_{\mathrm{c}}=A>1$ and $\mu_{\mathrm{c}}=1$ with the magnetic one having $\varepsilon_{\mathrm{c}}=1$ and $\mu_{\mathrm{c}}=A$, do not lead to a change of $\sigma$. Moreover, figure 8 shows that an anomalous (positive-valued) shift of the minima of $\sigma$ occurs if $\sqrt{\varepsilon_{\mathrm{s}}} \sqrt{\mu_{\mathrm{s}}}<0$ and $\varepsilon_{\mathrm{c}}$ is increased. For the comparison, two minima appear at $\varepsilon_{\mathrm{s}}=\mu_{\mathrm{s}}=5.8$ and $\varepsilon_{\mathrm{c}}=2.8$ within the same $k R$ range as in figure 8 . However, in this case $\sigma_{\min } \approx 3 \times 10^{-3}$ for the first minimum at $k R=1.8$, while $\sigma_{\min } \approx 0.18$ for the second minimum at $k R=3.52$. 


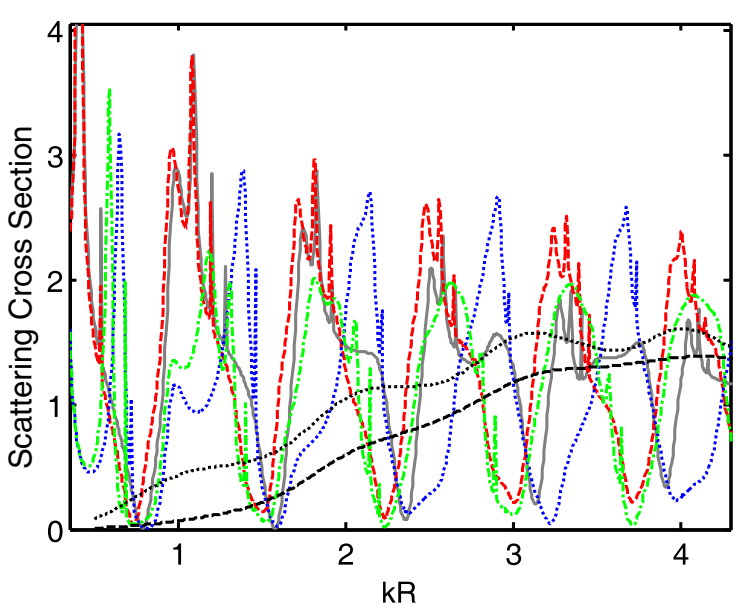

Figure 9. Scattering cross section versus $k R$ at $R / r=1.4$ : lines with multiple strong extrema- $\varepsilon_{\mathrm{s}}=35.4$ and $\mu_{\mathrm{s}}=5.8 ; \varepsilon_{\mathrm{c}}=1$, TM polarization (solid line); $\varepsilon_{\mathrm{c}}=2.8$, TM polarization (dashed line); $\varepsilon_{\mathrm{c}}=1$, TE polarization (dotted line); and $\varepsilon_{\mathrm{c}}=2.8$, TE polarization (dashed-dotted line); lines without multiple extrema- $\varepsilon_{\mathrm{s}}=\mu_{\mathrm{s}}=1$, $\varepsilon_{\mathrm{c}}=2.8$, TE polarization (dashed line) and TM polarization (dotted line); $\mu_{\mathrm{c}}=1$.

Therefore, only the first of them can be assigned to the masking regime. Similar to figure 4(a), the negative-valued shift of the minima occurs while increasing $\varepsilon_{\mathrm{c}}$.

\subsection{Dielectric object inside mismatched shell}

To obtain multiple frequencies, at which $\sigma \approx 0$, it is no longer necessary that $Z_{\mathrm{s}}=Z_{0}$. Figure 9 shows $\sigma$ versus $k R$ for the shell, which differs from that in figure 4(a) in smaller $\mu_{\mathrm{s}}$, while $\varepsilon_{\mathrm{s}}$ is kept and $Z_{\mathrm{s}} \neq Z_{0}$. Several minima of $\sigma$ do appear. It is interesting that, in some cases, $\sigma_{\min }$ at $\varepsilon_{\mathrm{c}}=2.8$ can be even closer to zero than that at $\varepsilon_{\mathrm{c}}=1$. The locations of the minima approximately satisfy (5). However, not all of them can be associated with the masking regime, because of $\sigma_{\min }$ and/or $\sigma_{\min } / \sigma^{\text {nc }}$ being relatively large. For example, for TE polarization in figure $9, \sigma_{\min }>\sigma^{\mathrm{nc}}$ at $k R=0.74$, while $\sigma_{\min } / \sigma^{\mathrm{nc}}<1 / 30$ and $\sigma_{\min }<0.04$ at $k R=2.24$ and 3.71 . In the latter case, $2 r / \lambda \approx 0.84$. As expected, an increase of $\varepsilon_{\mathrm{c}}$ at $\sqrt{\varepsilon_{\mathrm{s}}} \sqrt{\mu_{\mathrm{s}}}>0$ results in a shift of the minima towards smaller $k R$.

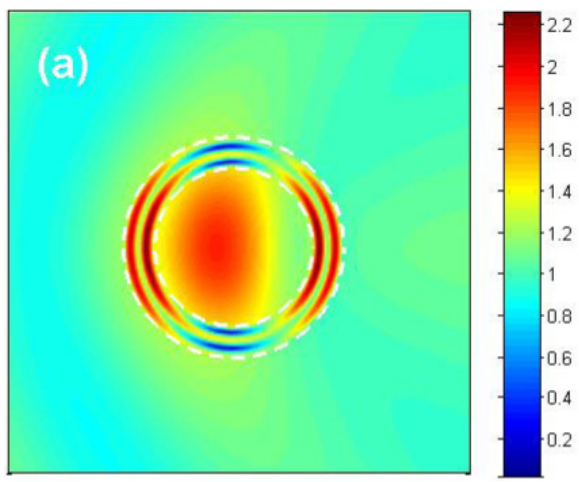

Figure 10 shows the typical near-field patterns at the minima of $\sigma$. They correspond to $\sigma \approx 0.06$ in plot (a) and $\sigma \approx 0.02$ in plot (b). The main difference between the cases of $Z_{\mathrm{s}} \neq Z_{0}$ and $Z_{\mathrm{s}}=Z_{0}$ is a larger $\left|H_{z}\right|$ within the shell in the former case. It is noteworthy that in figure 10(a) the slightly flattened field pattern in the core is shifted towards the smaller abscissa values. In contrast, in figure 10(b), the flattened pattern in the core is mainly localized near its center, while the field distribution in the shell is typical for a whisperinggallery mode. Strong topological differences between the fields in the core, shell and surrounding space demonstrate that the interpretation in terms of isolation can be used in the mismatched case, too. Note that $\sigma_{\min } / \sigma^{\mathrm{nc}} \approx 1 / 36$ for the $k R$ value in figure $10(\mathrm{~b})$.

The typical field patterns at the maxima of $\sigma$ in most cases also differ from those in the matched case. In particular, rather large values of $\left|H_{z}\right|$ can occur in the core. For example, at $k R=1.81, \varepsilon_{\mathrm{s}}=35.4, \mu_{\mathrm{s}}=5.8, \varepsilon_{\mathrm{c}}=2.8, \mu_{\mathrm{c}}=1$ and TE polarization, more than a fourfold enhancement of $\left|H_{z}\right|$ as compared to the incident wave is achieved within the core near the core-shell boundary at $\phi=0$ (large positive values of abscissa and zero ordinate), while the field in the adjacent subregion of the shell is also enhanced. At $k R=2.62$ and the other parameters the same, the field pattern looks like that of an asymmetric whispering-gallery mode, showing a rather strong penetration into the core within three subregions in the vicinity of $\phi=0$ and $\phi \approx \pm 0.37 \pi$. It follows from the obtained results that the localization of the field within the shell and/or the core subregion(s) being adjacent to the shell is a signature of the appearance of a strong scattering.

\subsection{Shell made of Drude-Lorentz material}

Let us now show how the effects studied in the previous sections for the hypothetical dispersion-free materials of the shell can manifest themselves for dispersive materials. Here, we restrict our consideration to the case when the material parameters of the shell depend on $\omega$ according to the DrudeLorentz model [12], i.e.

$$
\varepsilon_{\mathrm{s}}(\omega)=1-C \omega_{\mathrm{pe}}^{2} /\left(\omega^{2}-\omega_{0 \mathrm{e}}^{2}-\mathrm{i} \Gamma_{\mathrm{e}} \omega\right)
$$

and

$$
\mu_{\mathrm{s}}(\omega)=1-D \omega_{\mathrm{pm}}^{2} /\left(\omega^{2}-\omega_{0 \mathrm{~m}}^{2}-\mathrm{i} \Gamma_{\mathrm{m}} \omega\right)
$$

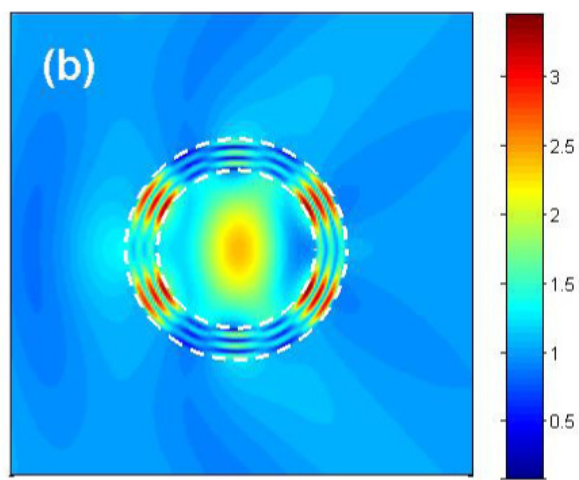

Figure 10. Axial magnetic field for TE polarization at $\varepsilon_{\mathrm{s}}=35.4, \mu_{\mathrm{s}}=5.8, \varepsilon_{\mathrm{c}}=2.8, \mu_{\mathrm{c}}=1, R / r=1.4$, and $k R=1.53$ (a) and $k R=2.24$ (b). 

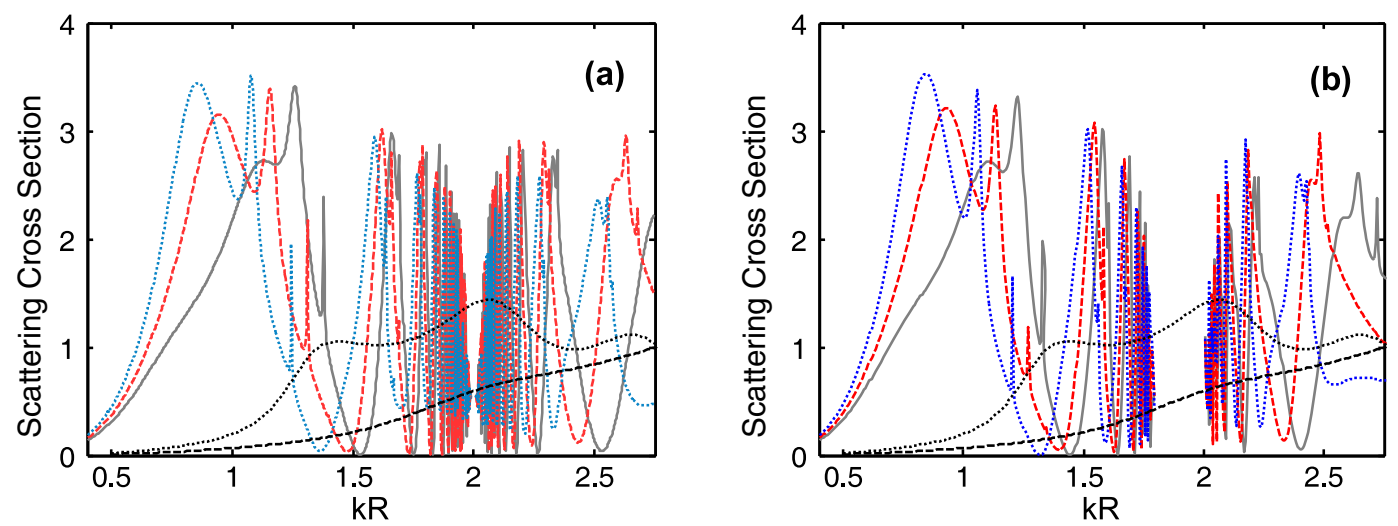

Figure 11. Scattering cross section versus $k R$ for TE polarization at $R / r=1.4$ and $\mu_{\mathrm{c}}=1$ : solid line $-\varepsilon_{\mathrm{c}}=1$, dashed lines $-\varepsilon_{\mathrm{c}}=2.8$ and dotted lines $-\varepsilon_{\mathrm{c}}=5.8$; lines with multiple extrema $-\Gamma_{\mathrm{e}} / \omega_{\mathrm{pe}}=10^{-4}$ and $C=D=1 ; \omega_{0 \mathrm{e}} R / c=2, \omega_{\mathrm{pe}} R / c=3.38, \omega_{0 \mathrm{~m}}=\omega_{0 \mathrm{e}}, \omega_{\mathrm{pm}}=\omega_{\mathrm{pe}}$ and $\Gamma_{\mathrm{m}}=\Gamma_{\mathrm{e}}-$ plot (a); $\omega_{0 \mathrm{e}} R / c=1.8, \omega_{\mathrm{pe}} R / c=3.21, \omega_{0 \mathrm{~m}} R / c=2, \omega_{\mathrm{pm}} R / c=3.38$ and $\Gamma_{\mathrm{m}} / \omega_{\mathrm{pm}}=10^{-4}$-plot (b); lines without multiple extrema- $\varepsilon_{\mathrm{s}}=\mu_{\mathrm{s}}=1$.

If $\omega_{0 \mathrm{e}}=\omega_{0 \mathrm{~m}}, \omega_{\mathrm{pe}}=\omega_{\mathrm{pm}}, \Gamma_{\mathrm{e}}=\Gamma_{\mathrm{m}}$ and $C=D$, one obtains $Z_{\mathrm{s}}=Z_{0}$ at all frequencies. Otherwise, the matching can occur simultaneously for two frequencies, at which $\varepsilon_{\mathrm{s}}(\omega)=\mu_{\mathrm{s}}(\omega)$. However, this is not necessary, as follows from the results of section 3.3 obtained for $\mu_{\mathrm{s}} \neq \varepsilon_{\mathrm{s}}$. According to equations (6) and (7), $\varepsilon_{\mathrm{s}}$ and $\mu_{\mathrm{s}}$ might take the values from a wide range of variation, while $k R$ varies slightly. Among them, several pairs of values of $\varepsilon_{\mathrm{s}}$ and $\mu_{\mathrm{s}}$ and, hence, several values of $\omega$ can be present, for which $\sigma \approx 0$.

Figure 11(a) presents the results for the shell, which is made of a low-loss dispersive material and matched with the surrounding free space. The multiple extrema appear so that the smaller the $\left|\omega-\omega_{0 \mathrm{e}}\right|$ the denser they are. This feature is in agreement with the results of sections 3.2 and 3.3, which are related to the effect of $\varepsilon_{\mathrm{s}}$ and $\mu_{\mathrm{s}}$ on the minima density. In this example, $\sigma \approx 0$ at several frequencies for $\varepsilon_{\mathrm{c}}=1$ and 2.8, while there is such a frequency for $\varepsilon_{\mathrm{c}}=5.8$. Due to the dispersion model used, all the minima with $\sigma \approx 0$ are now located in the vicinity of $\omega=\omega_{0 \mathrm{e}}$ and/or $\omega=\omega_{0 \mathrm{~m}}$. The general trend is that an increase of the number of minima is accompanied by their becoming more dense, which is connected with the increase of $\operatorname{Re} \varepsilon_{\mathrm{s}}$ and $\operatorname{Re} \mu_{\mathrm{s}}$. The extent to which the minima might become denser, depending on the model parameters in (6) and (7), is a subject of future studies.

Figure 11(b) shows $\sigma$ versus $k R$ for the shell made of a mismatched (with the exception of two frequencies, at which $\left.\varepsilon_{\mathrm{s}}(\omega)=\mu_{\mathrm{s}}(\omega)\right)$, low-loss, dispersive material. The same features are observed here as in figure 11(a). A difference is that now the range of ultra-high losses, for which $\sigma$ is not shown, is wider. In figure 11(b), $\sigma<0.05$ for $\varepsilon_{\mathrm{c}}=2.8$ at least at $k R=1.4,1.63,1.705$ and 1.74. In comparison, in figure $11(\mathrm{a}), \sigma<0.04$ for $\varepsilon_{\mathrm{c}}=2.8$ at least at $k R=1.476$, $1.732,1.867,1.894$ and 1.942 .

The realization of a multifrequency masking can be problematic if the losses are relatively high. In figure 12, the results are presented for the shell, which differs from that in figure 11(a) in stronger losses. In particular, the number of frequency values with $\sigma \approx 0$ is affected by the losses. Here, $\sigma<0.05$ at a sole $k R$ value for both $\varepsilon_{\mathrm{c}}=2.8(k R=1.475)$

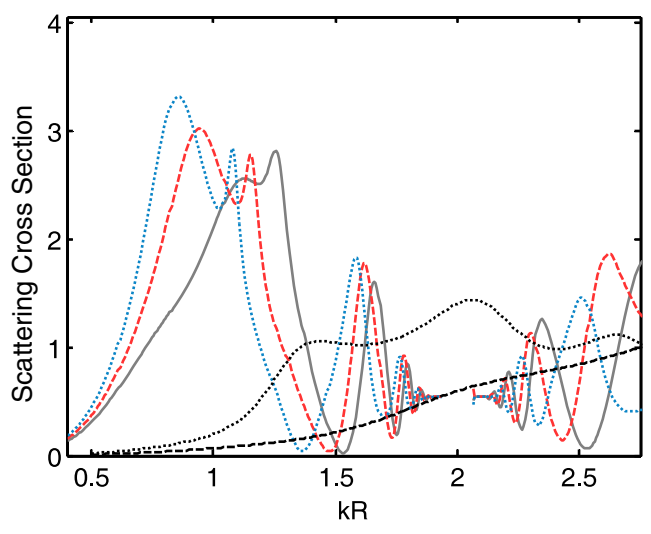

Figure 12. Same as figure 11(a) but for $\Gamma_{\mathrm{e}} / \omega_{\mathrm{pe}}=10^{-2}$.

and $\varepsilon_{\mathrm{c}}=5.8(k R=1.365)$. Note that these minima remain nearly at the same locations as in figure 11(a). Comparing figure 11 with figure 12 , one can see how large the losses might be for obtaining $\sigma \approx 0$ at multiple frequencies. At the same time, the obtained results show that a single-frequency masking can be obtained even at relatively high losses.

\section{Conclusions}

The potential of single-layer shells, which are made of isotropic metamaterials with simultaneously large positive or large negative permittivity and permeability, in multifrequency reduction of the scattering cross section of dielectric cylinders has been studied. The most interesting observed regimes can be assigned to non-ideal cloaking or weak-scattering masking. They can be obtained for a wide range of parameter variation, including that corresponding to the resonant-sized cylinders. The exploited physical mechanism is related to the halfwavelength Fabry-Perot-type radial resonances, which appear within the shell. The number and density of the resonance frequencies determine those of the minima and maxima of the scattering cross section. The larger/smaller positive/negative index of refraction of the shell material and the shell thickness, 
the larger the number of minima with near-zero scattering cross section that could exist. At the minima, the field inside the core usually shows rather large values and a rather strong dependence on the coordinates. The effects studied for the hypothetical dispersion-free materials have been validated for the shells, which are made of materials with DrudeLorentz dispersion. In this case, both double-positive and double-negative regimes can be simultaneously involved in the multifrequency cloaking. Similar effects are expected to appear for other types of dispersion.

\section{Acknowledgments}

This work is supported by the European Union under the projects EU-PHOME and EU-ECONAM, and TUBITAK under project nos. 105A005, 106E198 and 107A004. The authors acknowledge support from the Turkish Academy of Sciences and the TUBITAK-2221 program.

\section{References}

[1] Nicorovici N A P, Milton G W, McPhedran R C and Botten L C 2007 Opt. Express 156314

[2] Milton G W and Nicorovici N P A 2006 Proc. R. Soc. A 4623027

[3] Milton G W, Nicorovici N P A and McPhedran R C 2007 Physica B 394171

[4] Pendry J B, Schurig D and Smith D R 2006 Science 3121780

[5] Schurig D, Mock J J, Justice B J, Cummer S A, Pendry J B, Starr A F and Smith D R 2007 Science 314977

[6] Alu A and Engheta N 2005 Phys. Rev. E 72016623

[7] Alu A and Engheta N 2007 Opt. Express 153318

[8] Leonhardt U 2006 Science 3121777

[9] Cai W, Chettiar U K, Kildishev A V and Shalaev V M 2007 Nat. Photon. 1224

[10] Xiao D and Johnson H T 2008 Opt. Lett. 33860
[11] Katsarakis N, Konstantinidis G, Kostopoulos A, Penciu R S, Gundogdu T F, Kafesaki M, Economou E N, Koschny Th and Soukoulis C M 2005 Opt. Lett. 301348

[12] Soukoulis C M, Economou E N and Kafesaki M 2006 Adv. Mater. 181941

[13] Linden S, Enkrich C, Dolling G, Klein M W, Zhou J, Koschny Th, Soukoulis C M, Burger S, Schmidt F and Wegener M 2006 IEEE J. Sel. Top. Quantum Electron. 121097

[14] Dolling G, Enkrich C, Wegener M, Soukoulis C M and Linden S 2006 Opt. Lett. 311800

[15] Urzhumov Y A and Shvets G 2008 Solid State Commun. 146208

[16] Li T, Liu H, Wang F M, Li J Q, Zhu Y Y and Zhu S N 2007 Phys. Rev. E 76016606

[17] Cai W, Chettiar U K, Kildishev A V and Shalaev V M 2008 Opt. Express 165444

[18] Kante B, de Lustrac A, Lourtioz J M and Burokur S N 2008 Opt. Express 169191

[19] Huang Y, Feng Y and Jiang T 2007 Opt. Express 1511133

[20] Smolyaninov I I, Hung Y J and Davis C C 2008 Opt. Lett. 331342

[21] Alu A and Engheta N 2008 Phys. Rev. Lett. 100113901

[22] Alu A and Engheta N 2008 Electromagnetics 28464

[23] Teixeira F L 2007 IEEE Antennas Wirel. Propag. Lett. 6163

[24] Ma Y G, Liu Y, Tan C Y, Liu Z W and Ong C K 2006 J. Appl. Phys. 100054307

[25] Wu L Z, Ding J, Neo C P, Chen L F and Ong C K 2007 Phys. Status Solidi a 204755

[26] Lepetit T and Akmansoy E 2007 Magnetism in high-contrast dielectric photonic crystals Proc. 2nd Europ. Conf. Antennas Propag. (Edinburgh, Nov. 2007)

[27] Jylhä L, Kolmakov I, Maslovski S and Tretyakov S 2006 J. Appl. Phys. 99043102

[28] Pimenov A and Loidl A 2006 Phys. Rev. B 74193102

[29] Shvets G and Urzhumov Y A 2005 J. Opt. A: Pure Appl. Opt. $7 \mathrm{~S} 23$

[30] Wu G and Park W 2008 Appl. Phys. Lett. 92153114

[31] Podolskiy V A and Narimanov E E 2005 Phys. Rev. B 71201101 